\title{
The design and structural analysis of the endoprosthesis of the shoulder joint
}

\author{
Robert Karpiński ${ }^{1}$, Łukasz Jaworski ${ }^{2}$, Jarosław Zubrzycki ${ }^{1, *}$ \\ ${ }^{1}$ Lublin University of Technology, Faculty of Mechanical Engineering, Department of Machine Design and Mechatronics, \\ Nadbystrzycka 36, 20-618, Lublin, Poland \\ ${ }^{2}$ Poznan University of Technology, Faculty of Mechanical Engineering and Management, 60-965 Poznań, Poland
}

\begin{abstract}
The shoulder joint is a crucial element of the upper limb and is necessary to maintain full mobility during daily activities. Similar as in the hip, it is an example of a ball and socket joint, enabling articulation between the head of the humerus and the glenoid cavity of the scapula. By studying kinematics of upper limbs, it is possible to distinct several possible movements in the shoulder joint. Due to many painful diseases and medical conditions, it may be crucial to perform a pain reducing procedure, like shoulder joint replacement. In order to preserve the function of the joint, the endoprosthesis should be designed exclusively for the patient. To assess a scale of damage in the joint or its specific structure, the shoulder was scanned using the computed tomography procedure. Results of the scan were processed with the use of Materialise Mimics software, which converts standard 2D images into 3D CAD models. Necessary analysis and measurements were taken leading to the beginning of the designing process. The prosthesis was created using Solid Edge software, developed especially for the purpose of rapid prototyping. After determining the physical properties of structural materials, the Finite Elements Analysis of the model was conducted using SolidWorks Simulation software under various load conditions.
\end{abstract}

\section{Introduction}

The shoulder joint is a crucial element of the upper limb and is necessary to maintain full mobility during daily activities. Unfortunately, due to pathological changes in the joint, it is often partially or completely impossible to fully exploit its capabilities. The ageing of the population, as a cause of growing demand for medical services dedicated to older people, poses a challenge to engineers trying to design solutions fully corresponding with the demand from both patients and physicians, especially in orthopaedics, and endoprosthesis in particular.

\section{The anatomy of a shoulder joint}

A shoulder girdle is connected with the upper limb (arm) via the shoulder joint (or glenohumeral joint - articulatio humeri) shown on Figure 1. The glenohumeral joint is a multiaxial, synovial ball and socket joint, one of the largest joints of this type in the human body. It guarantees movements such as flexion and extension, abduction and adduction, lateral and medial rotation, horizontal abduction and adduction, and a combination of mentioned movements called circumdiction. The articular surfaces of the joint consist of the head of the humerus and the glenoid cavity of the scapula. The head of the humerus is a section of a ball, and its surface is almost four times larger than the surface of an acetabulum (socket) of the joint. The surface of the socket is slightly recessed, oval-shaped and perpendicular to the surface of a scapula. Articular surfaces are covered with hyaline cartilage, which on the peripheries of the socket transforms into a few (up to 6) millimetres thick fibrocartilaginous rim called a glenoid labrum. The articular cartilage transfers and spreads loads between bones, while preserving appropriate stress distribution on the surface of the joint, reduces friction, absorbs sudden overstrains and protects bones from surface wear. Fully developed cartilage does not contain blood vessels or nerves. A very loose connection between the socket and the ball, along with a strong and large articular capsule, guarantees great mobility in the joint $[1,2,3,4,5]$.

Beside muscles and tendons strengthening the joint capsule, there are also articular ligaments stabilizing the shoulder joint, such as coracohumeral ligament, glenohumeral ligaments and coraco-acromial ligament $[1,2,3]$.

Coracohumeral ligament is the strongest ligament in the shoulder joint, and is located on the upper surface of the joint, between a coracoid process and the lesser and greater tuberosities of the humerus. Glenohumeral ligaments are located on the anterior side of the glenohumeral joint; the coraco-acromial ligament does not directly participate in strengthening the joint, but plays a crucial part in its biomechanics and protection $[1$, 2].

\footnotetext{
* Corresponding author: robert.karpinski@pollub.edu.pl
} 


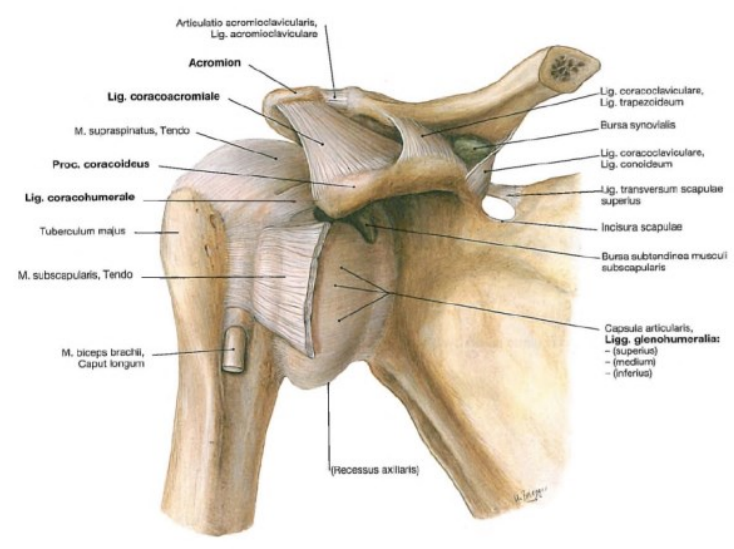

Fig. 1. Right shoulder joint (front view) [2].

\section{Replacement of the shoulder joint}

Alloplasty is a procedure that consists of implementing elements acting as a replacement for damaged parts of a joint into the human body, which can reinstate lost functions to these parts of the body. An endoprosthesis is an implant made of biocompatible materials, which acts as a replacement for damaged joints. Alloplasty of the shoulder joints, thanks to the implementation of the endoprosthesis in place of the defective joint, allows radically reducing pathological changes and pain, as well as recreating functions of the shoulder $[4,5,6]$.

Endoprosthetics of the shoulder joint, or the shoulder joint replacement, is mainly performed on patients with osteoarthritis. Osteoarthritis is a group of overlapping medical conditions, which in spite of having different etiologies; cause similar biological, morphological and medical effects. A disease process affects not only the articular cartilage, but also the joint itself, including the subchondral bone, ligaments, the joint capsule, a synovial membrane and muscles around the joint. The osteoarthritis is one of the most frequently occurring diseases of the motion system, especially with elder people. It is often caused by biological and mechanical occurrences disordering a synthesis and triggering the defeneration of the articular cartilage, in chondrocytes and an extracellular matrix as well as in the subchondral layer of the bone $[5,6,7,8]$.

There are several conditions causing limitations in the mobility of the shoulder joint, including [7, 9]:

- changes in an acromioclavicular and a sternoclavicular joint, effecting in the limitation of movements of the scapula and a collarbone,

- inflammation of synovial bursae,

- weakening of muscles of the shoulder girdle,

- rotator cuff damage,

- increase of muscle tension, due to the pain in particular,

- constriction of the articular capsule,

- articular cartilage damage, effecting in a deficiency of a slide movement in the joint.

\section{The design of the endoprosthesis of the shoulder joint}

\subsection{Software tools used in the study}

Materialise Mimics allows the user to process and edit 2D images from CT or MRI scans in order to generate highly accurate 3D models. Moreover, it is possible to make precise measurements (dimensions, density) of bones, tissues or organs. The patient-specific models can be implemented in a variety of engineering applications directly in Materialise Mimics or Materialise 3-matic, or exported to 3D models and anatomical landmark points to third party software, like statistical, CAD, or FEA packages [10].

Solid Edge ST is a complex, parametric, hybrid 2D/3D CAD system using synchronous technology. Implementation of two highly-efficient graphic modellers - Parasolid and D-Cubed allowed to combine direct modelling with precise control of geometry, which gives engineers the opportunity to conduct the process of designing with speed and simplicity on a level that has never been seen before. It provides tools for solid modelling, assembly modelling and 2D orthographic view functionality for mechanical designers. Through third party applications it has links to many other Product Lifecycle Management technologies [10].

\subsection{The design process}

The alloplasty of each ball and socket joint, such as the shoulder joint, requires the best possible fitting of the implant within anatomical structures of the patient's body. Due to the increasing precision of medical imaging methods, processing of given data and many features of the CAD software, it is possible to design the prosthetic implant individually fitted for the patient.

Probably the most important step in a process of designing the endoprosthesis is obtaining measurements of structures that are going to be replaced. Based on 3D models of a patient's shoulder girdle, it was possible to find approximate measurements of the glenoid cavity of the scapula (length, width - Fig. 2) and the head of the humerus (diameter, angle between the axis of the head and axis of the shaft etc. - Fig. 3). Additionally, a parameter was determined on how much of the glenoid cavity could be excised to properly mount the implant.

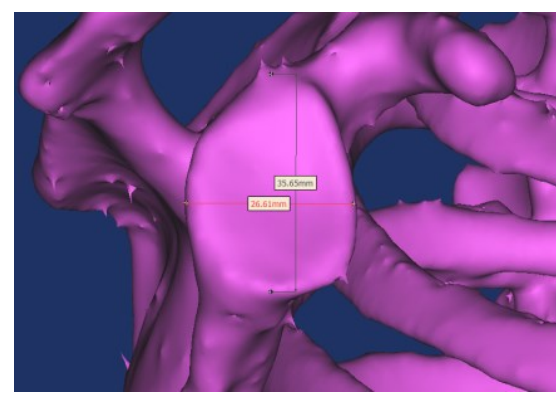

Fig. 2. Measurements of the glenoid cavity of the scapula. 


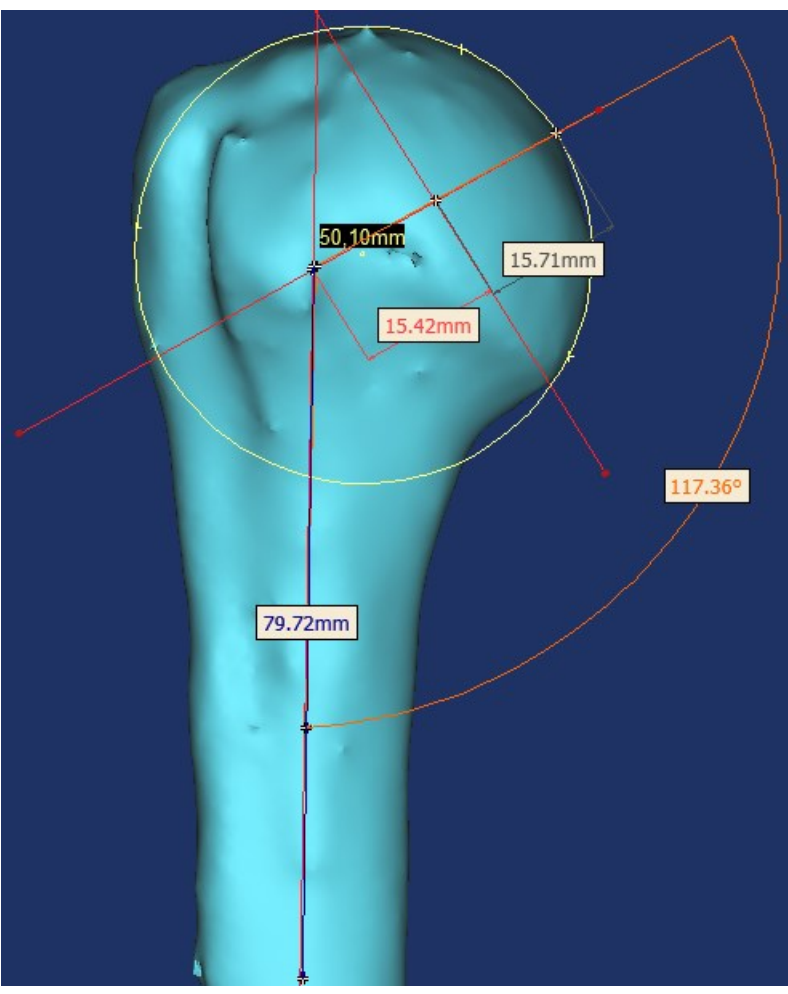

Fig. 3. Measurements of the humerus.

These values became a base for designing actual elements: a shell - replacing the glenoid cavity, a cup acting as the articular cartilage, and the head on the shaft - substituting the top part of the humerus.

The shell and the cup (Fig. 4) are shaped like a section of a sphere. The shell is supposed to be mounted to the dissected articular cavity using a $\varnothing 4.5 \mathrm{~mm}$ orthopaedic screw and is stabilized by two pins. The cup is designed to tightly fit the shell. Its inner diameter (ID) is $45 \mathrm{~mm}$ while the outer diameter (OD) is $60 \mathrm{~mm}$, which leads to an overall thickness of the set equal to $7.5 \mathrm{~mm}$. The length and the width are respectively $35 \mathrm{~mm}$ and $25 \mathrm{~mm}$, equivalent to measured values.

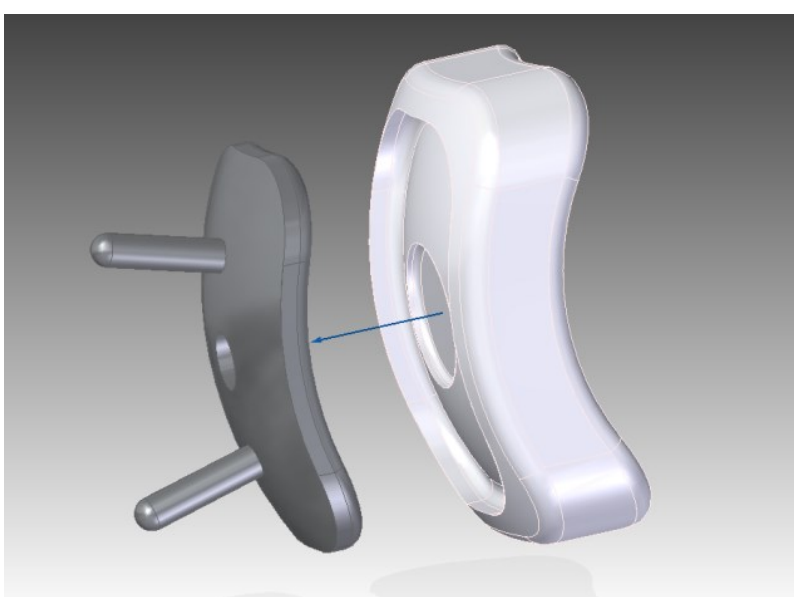

Fig. 4. The shell (left) and the cup (right) of the endoprosthesis.

The humerus' part of the endoprosthesis (Fig. 5) consists of the spherical cap shaped head and the shaft. The diameter of the head matches inner diameter of the cup, making it possible to perform expected movements. The shaft (or stem) is the element directly inserted into the humerus, hence the specific shape tampering to the downside with additional protrusions on the surface to ensure stability of the stem and maximize the process of bone healing, considering that the planned operation is going to be performed without the use of bone cement. The head is tightly mounted on the neck of the shaft with a mounting hole and is prevented from rotating with a protrusion on the neck matching an indentation in the mounting hole.

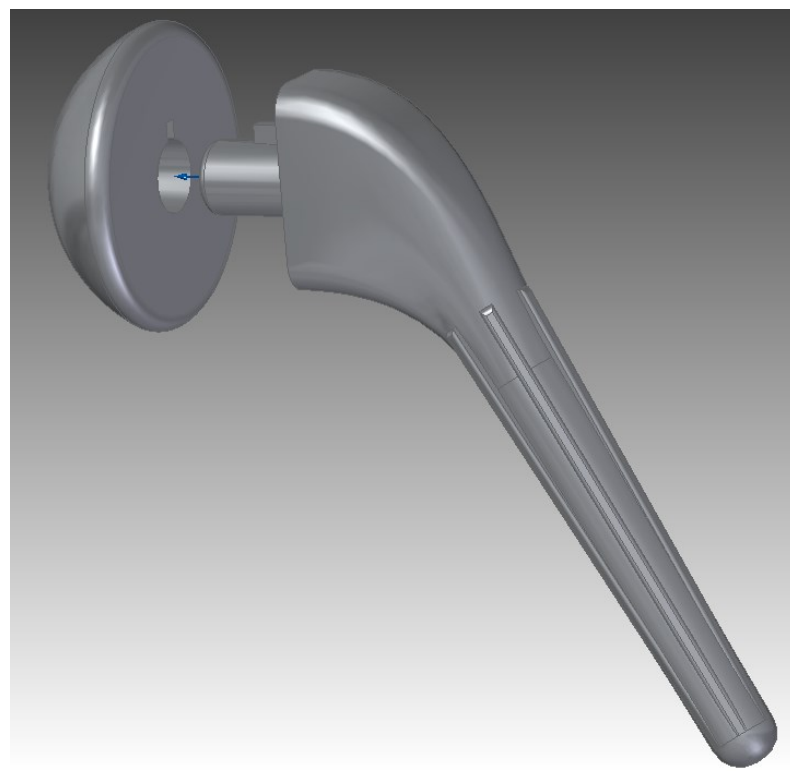

Fig. 5. The head and the shaft of the endoprosthesis.

\section{Study on stress distribution in the endoprosthesis}

The designed model was used to perform a series of preliminary studies including stress distribution in the endoprosthesis with the use of Finite Element Analysis method.

\subsection{FEA method}

Finite Element Analysis is one of the basic methods of conducting computer aided engineering calculations. It is one of the techniques of discretization of geometric systems, i.e. dividing a continuum into a finite amount of subareas.

The main principle of FEA is to divide geometric models into elements uniting in nodes, which effects in creating a discrete geometric model, split in simply shaped subareas, called the finite elements. During performing of calculations with the use of FEA, other physical quantities are being discretized, like loads, tensions, restraints or other examples represented in the system with the use of continuous function. While performing the process of discretization the software aims at maximally approximation of discreet and continuous form using approximation methods. After converting the data analysis follows, consisting in uniting individual 
elements as a whole using equilibrium conditions and displacement compatibilities, which results in receiving a set of algebraic, simultaneous equations, posing as a mathematical description of the analysed problem. Afterwards, the mentioned equations are being solved using values of equilibrium conditions, and their outcome used to compute sought quantities, i.e. tensions.

\subsection{Methodology of studies}

Studies were performed in the environment of Solidworks Simulation, which provides core simulation tools to test designs and to make the decisions to improve their quality. Full integration creates a short learning curve and eliminates the redundant tasks required with traditional analysis tools. Component materials, connections, and relationships defined during design development are fully understood for simulation. Its main advantage is its cost, which is much lower than any other FEA software. In order to achieve credible results, materials (of which the element would be made) were assigned to each element. Properties of materials are presented in Table 1.

Table 1. Table of materials

\begin{tabular}{|c|c|c|c|}
\hline Material & Element & $\begin{array}{c}\text { Young's } \\
\text { modulus } \\
{[\mathrm{MPa}]}\end{array}$ & $\begin{array}{c}\text { Poisson's } \\
\text { ratio [-] }\end{array}$ \\
\hline $\begin{array}{c}\text { Titanium alloy } \\
\text { Protasul Ti }\end{array}$ & $\begin{array}{c}\text { Prosthetic } \\
\text { shell }\end{array}$ & 110000 & 0.3 \\
\hline $\begin{array}{c}\text { Titanium alloy } \\
\text { Protasul 100 }\end{array}$ & Shaft, head & 100000 & 0.3 \\
\hline Polyethylene & $\begin{array}{c}\text { Socket inlay } \\
\text { (cup) }\end{array}$ & 1070 & 0.41 \\
\hline
\end{tabular}

The force was applied to the shaft of the endoprosthesis with values matching the value of the body weight (measured in the unit of Newton [N]) while performing different activities. In performed studies, the value of the human body weight was set on $784.8 \mathrm{~N}$ (or $80 \mathrm{~kg}$ ), which was then assumed as $100 \%$ of body weight (\%BW). Three possible scenarios were considered with the upper limb in abduction to $30^{\circ}, 65^{\circ}$ and $90^{\circ}$, where reaction forces influencing the endoprosthesis would have approximate values of, respectively, 30, 50 and $60 \% \mathrm{BW}[11]$.

\subsection{Results}

The analyses of the simulations resulted in obtaining maps of stress distribution in elements of the endoprosthesis, shown below.

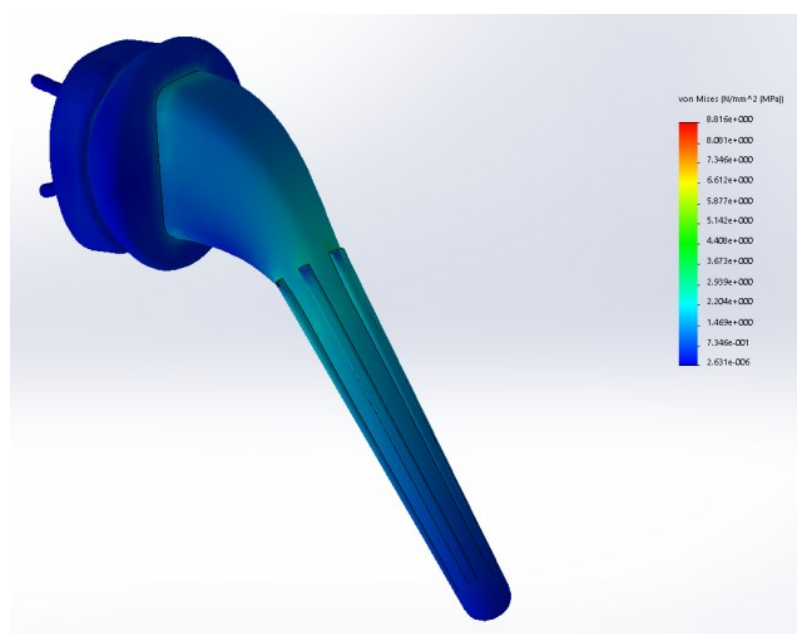

Fig. 6. Stress distribution in the endoprosthesis in $30^{\circ}$ abduction.

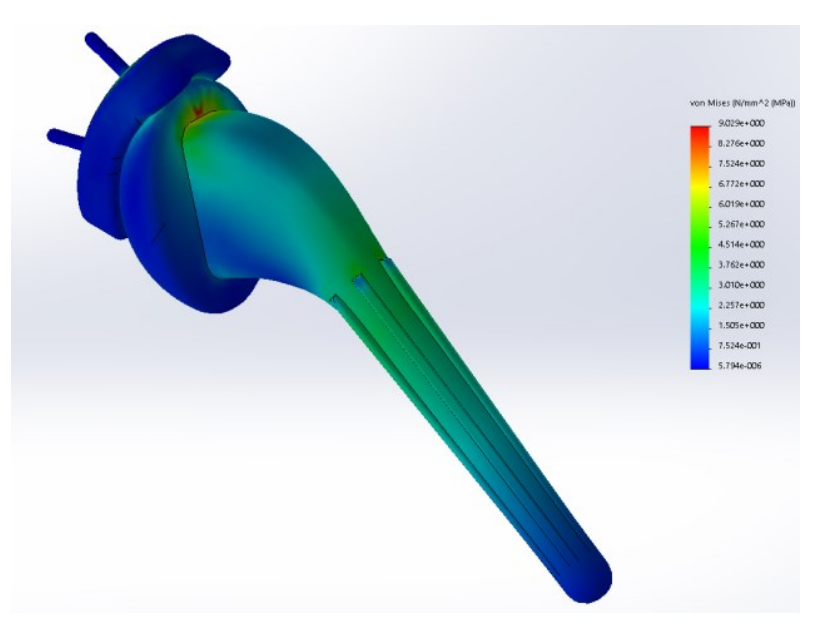

Fig. 7. Stress distribution in the endoprosthesis in $65^{\circ}$ abduction.

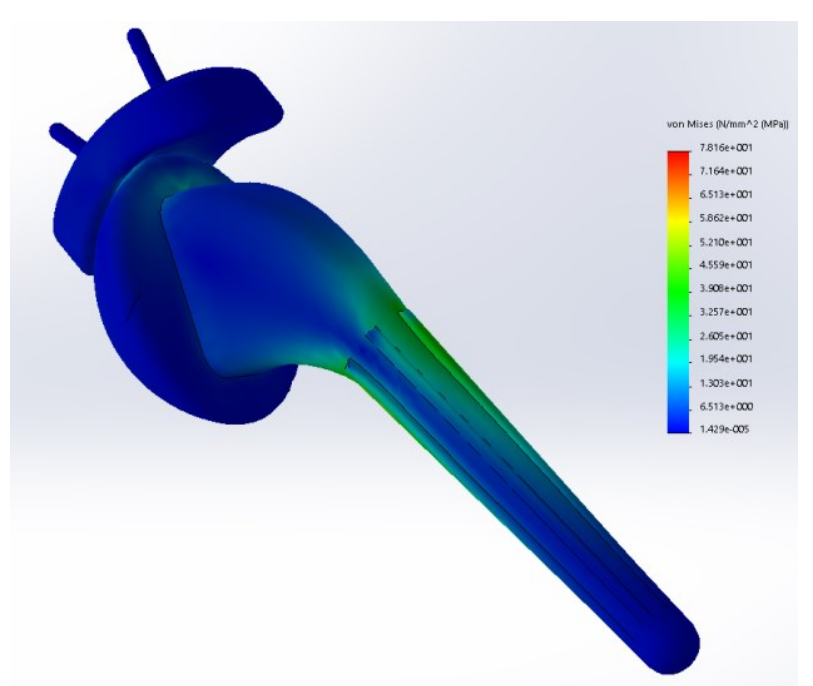

Fig. 8. Stress distribution in the endoprosthesis in $95^{\circ}$ abduction. 


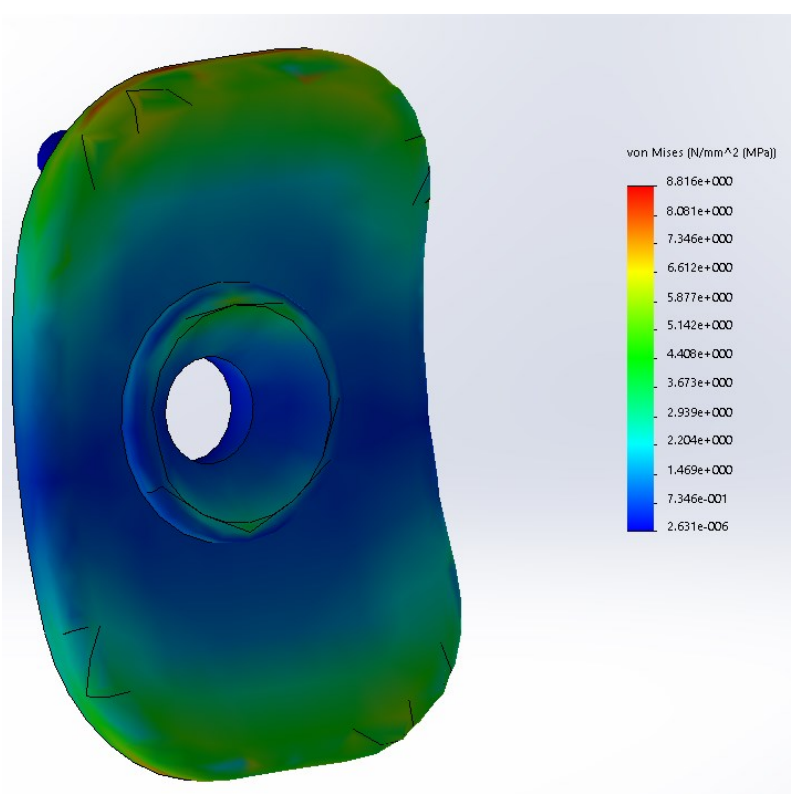

Fig. 9. Stress distribution in the prosthetic shell in $30^{\circ}$ abduction.

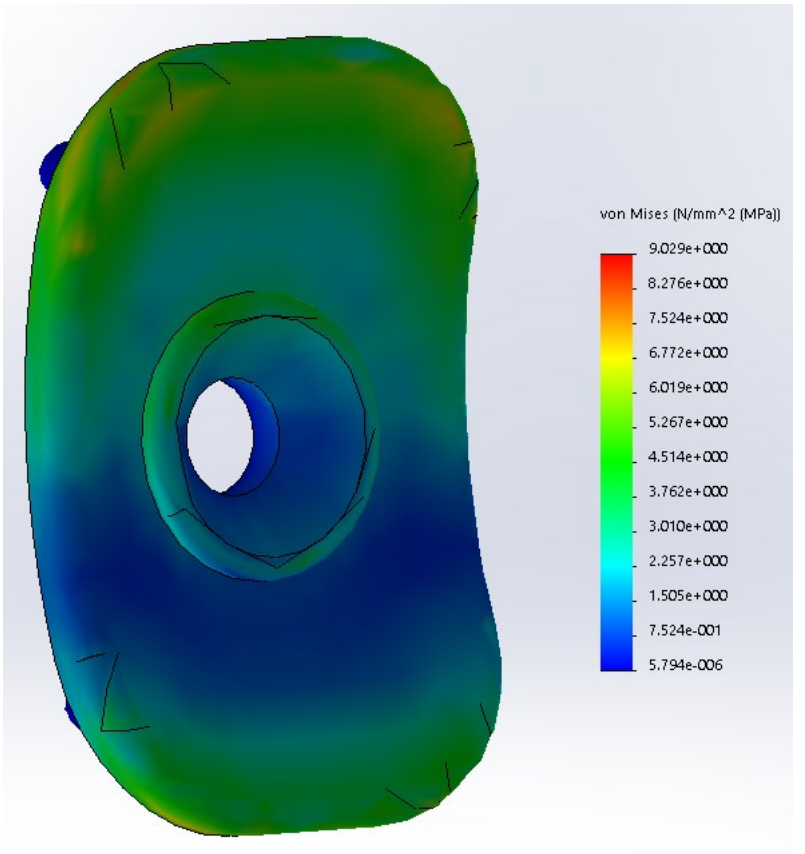

Fig. 10. Stress distribution in the prosthetic shell in $65^{\circ}$ abduction.

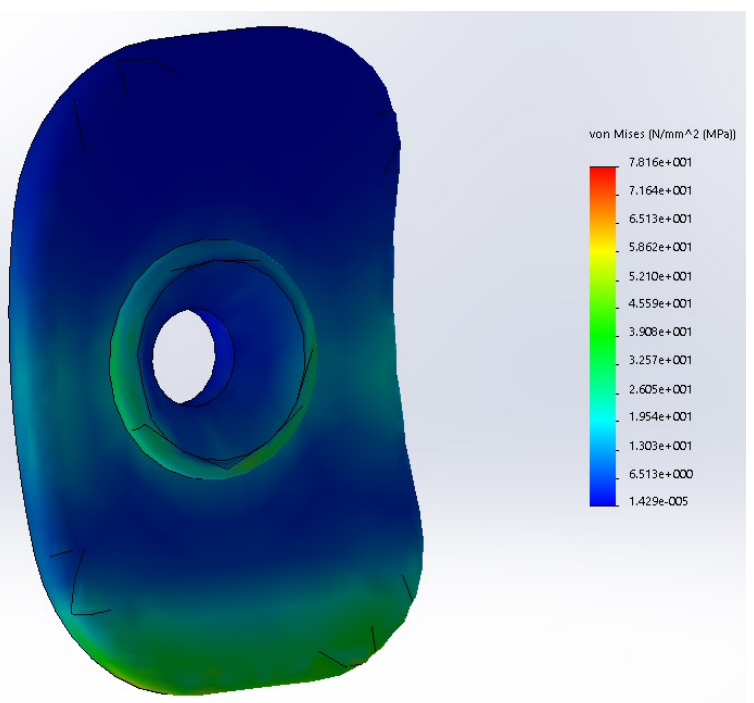

Fig. 11. Stress distribution in the prosthetic shell in $95^{\circ}$ abduction.

Performed analyses show that forces acting upon the endoprosthesis lead to different stress distributions depending on the value of the force and orientation of the prosthesis. As expected, the maximum value of von Mises stress, in each case, is located on the shaft of the endoprosthesis, mostly near protrusions.

More interesting is the stress distribution in the prosthetic shell, since it is more likely to be damaged because of its structure. In each scenario simulation indicated, that the applied force would have greater impact on the edges of the shell. With the change in the angle of abduction of the endoprosthesis, the stress distribution changes - in $30^{\circ}$ abduction the force acts almost evenly on the top and bottom edges of the shell, in $65^{\circ}$ it influences both edges along the upper half of the shell, while in $95^{\circ}$ most of the stress is located near the lower edge with small changes in the centre of the shell, which may indicate the possibility of the bending of the shell of the endoprosthesis.

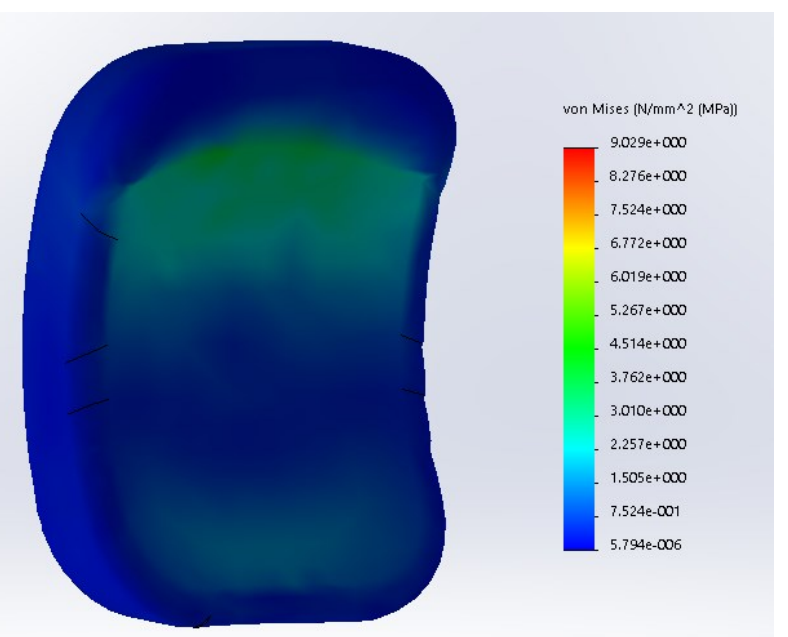

Fig. 12. Stress distribution in the socket inlay in $65^{\circ}$ abduction. 


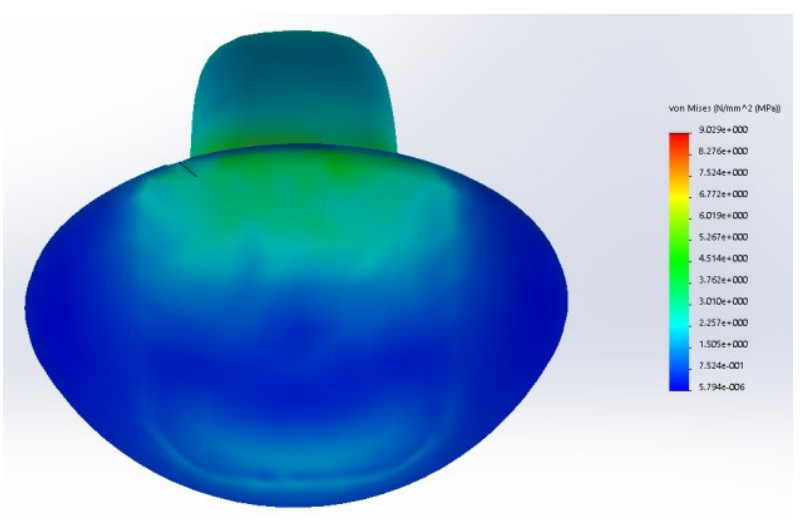

Fig. 13. Stress distribution in the head of the endoprosthesis in $65^{\circ}$ abduction.

In the case of the $65^{\circ}$ abduction it is distinctive that most of the force affecting the socket inlay (or the cup) accumulates in the area between the centre and the top edge, as seen in Fig. 12. The corresponding area on the head of the prosthesis, acting upon the socket inlay, is shown in Fig. 13.

\section{Conclusions}

Modern technology gives engineers and physicians tools to implement solutions designed solely for the purpose of saving or at least easing the life of the specific patient. Due to the fact that dimensions of the endoprosthesis are based on a patient's physiognomy, it is possible to precisely plan and perform the implantation procedure. Thus, introducing more individual ways of treating the patient could lead not only to a decrease of the time required for convalescence, but in effect also reducing overall costs, mostly associated with the hospitalization of the patient.

Implementation of the FEA simulations is a crucial part of the designing process, since it is possible to predict and counteract possible damages, which may occur as a result of the forces influencing the part. Based on the performed simulations for designed endoprosthesis it can be assumed, that under given loads the elements of the endoprosthesis would maintain their structural integrity since the elastic limit (or yield strength) wasn't exceeded. For more accurate data it would be beneficial to perform a series of studies on the properly manufactured endoprosthesis.

\section{References}

1. Z. Ignasiak, Anatomia układu ruchu (Elsevier Urban \& Partner, Wrocław, 2007)

2. A. Kontaxis, G. R Johnson, Clin. Biomech. 24, 254260 (2009)

3. W. Woźniak, K.S. Jędrzejewski, Sobotta. Atlas anatomii człowieka (Elsevier Urban \& Partner, Wrocław, 2012)

4. C.S. Neer, K.C. Watson, F.J. Stanton, . J. Bone Joint Surg. Am. 64 (3), 319-337 (1982)

5. R. Karpiński, Ł. Jaworski, J. Zubrzycki, Adv. Sci. Technol. Res. J. 10, 240-246 (2016)

6. R. Będziński, Biomechanika Inżynierska (Oficyna Wydawnicza Politechniki Wrocławskiej, Wrocław, 1997)

7. T.S. Gaździk, Biomechanika, 641-664 (IPPT PAN, Warszawa, 2011)

8. P.A Klimiuk, A. Kuryliszyn-Moskal, Reumatologia 50, 162-165 (2012)

9. A. Prusinowska, P. Turski, E. Przepiórski, P. Małdyk, K. Księżopolska-Orłowska, Reumatologia 51, 108-112

10. R. Karpiński, Ł. Jaworski, J. Szabelski, Appl. Comput. Sci. 12, 87-95 (2016)

11. P. Wodarski, R. Michnik, J. Jurkojć, A. Bieniek, M. Gzik, Modelowanie Inżynierskie, 29, 74-79 (2016 